Research Paper

\title{
In vitro Cytotoxic Activities of the Oral Platinum(IV) Prodrug Oxoplatin and HSP90 Inhibitor Ganetespib against a Panel of Gastric Cancer Cell Lines
}

\author{
Lukas Klameth², Barbara Rath², Gerhard Hamilton ${ }^{2} \bowtie$ \\ 1. Department for Pathophysiology and Allergy Research, Medical University of Vienna, Vienna, Austria \\ 2. Department of Surgery, Medical University of Vienna, Vienna, Austria \\ $\triangle$ Corresponding author: Hamilton Gerhard, Department of Surgery, Medical University of Vienna, A-1090 Vienna, Austria. Email: \\ gerhard.hamilton@meduniwien.ac.at \\ (c) Ivyspring International Publisher. This is an open access article distributed under the terms of the Creative Commons Attribution (CC BY-NC) license \\ (https://creativecommons.org/licenses/by-nc/4.0/). See http://ivyspring.com/terms for full terms and conditions.
}

Received: 2016.10.04; Accepted: 2017.04.01; Published: 2017.07.01

\begin{abstract}
Gastric cancer exhibits a poor prognosis and is the third most common cause of cancer death worldwide. Chemotherapy of metastatic gastric cancer is based on combinations of platinum drugs and fluoropyrimidines, with added agents. Oxoplatin is a stable oral platinum(IV) prodrug which is converted to a highly active tetrachlorido(IV) complex under acidic conditions. In the present work, we studied the cytotoxic effects of oxoplatin against a panel of four gastric cancer cell lines in vitro. Furthermore, the role of HSP90 in chemoresistance of these lines was investigated using the specific inhibitor ganetespib. The KATO-III, MKN-1, MKN-28, MKN-45 lines were used in MTT chemosensitivity, cell cycle and apoptosis assays. KATO-III is a signet ring diffuse cell type, $\mathrm{MKN}-1$ an adenosquamous primary, MKN-28 a well-differentiated intestinal type and the MKN-45 a poorly differentiated, diffuse type gastric carcinoma line. Cytotoxicity was tested in MTT assays and intracellular signal transduction with proteome profiler Western blot arrays. Interactions of platinum drugs and ganetespib were calculated with help of the Chou-Talalay method. The prodrug oxoplatin revealed low activity against the four gastric cancer cell lines, whereas the platinum tetrachlorido(IV) complex and cisplatin gave $I_{50}$ values of $1-3 \mu \mathrm{g} / \mathrm{ml}$ with increasing chemoresistance observed in the order of MKN-1, KATO-III, MKN-28 to MKN-45. With exception of KATO-III and MKN-28/oxoplatin, all other cell lines featured marked synergistic toxicity with clinically achievable concentrations of ganetespib. Oral administration of a platinum agent such as oxoplatin would be of great value for patients and care providers alike. These results suggest that the oncogene-stabilizing HSP90 chaperone represents an important mediator of chemoresistance in gastric cancer. Ganetespib reduced the phosphorylation of $p 53, A k t 1 / 2 / 3$ and PRAS40, as well as of WNK1, a kinase which regulates intracellular chloride concentrations. Intracellular chloride was reported to control proliferation of gastric cancer cell lines. Expression of $\mathrm{MUCl}$ was not downregulated in contrast to the expression of CAIX, a prognostic marker in gastric cancer. In conclusion, the HSP90 inhibitor ganetespib synergizes with platinum anticancer drugs and modulates intracellular signal transduction in direction of a less proliferative and aggressive phenotype.
\end{abstract}

Key words: Gastric cancer, HSP90, platinum cytostatics, chloride channel, ganetespib

\section{Introduction}

According to the GLOBOCAN 2012, estimated 952,000 new gastric cancer cases occurred worldwide in 2012. This makes stomach cancer the fifth most common cancer in the world after lung, breast, colorectal and prostate cancer and ranks third of the cancer-related deaths (approximately 723,000) [1]. 
Gastric cancer is especially prevalent in East Asia, Eastern Europe, and parts of Central America and South America [2]. The mortality rates of gastric cancer are declining over the last decades, mainly through the reduction of chemically preserved foods and life style changes. However, Helicobacter pylori infections remain the major risk factor for developing gastric cancer [3, 4]. This malignancy can be divided into intestinal and diffuse subtypes according to Lauren's classification. Most of all the gastric cancer cases will have sporadically acquired genetic alterations concerning TP53, PIK3CA, ARID1A, FAT4, MLL and MLL3 among others [5].

Surgical resection remains the first choice of treatment for gastric cancer, if the tumor is still regionally confined [6]. However, different studies revealed that neoadjuvant and/or adjuvant chemotherapy employing drugs such as epirubicin, cisplatin (cis-diammine-dichlorido-platinum(II)) and 5-fluorouracil (5-FU) significantly increased the 5-year survival compared to surgery alone [7]. In cases with unresectable and widespread tumors, administration of chemotherapy demonstrated a significantly benefit over best supportive care and, furthermore, combination chemotherapy yielded an increased survival outcome compared to a single agent regimen [8]. Targeted therapy with trastuzumab, a monoclonal antibody targeting HER2, and ramucirumab, a humanized monoclonal antibody targeting VEGFR-2 receptor, gave promising results for second-line therapy but may be not available commonly. Platinum(IV) based substances, like oxoplatin (cis, cis,trans-diaminodichlorido-dihydroxido platinum (IV)), are of particular interest, because of an increased inertness and the possibility of oral administration. The reduced reactivity of such platinum derivatives results in an increased half-live in the circulation and, therefore, fewer side-effects concurrent with high cytotoxicity in cisplatin-resistant cells $[10,11]$. Oxoplatin is converted to cis-diamminetetrachlorido-platinum(IV) (DATCP[IV]), which exhibits at least two-fold increased cytotoxic activity.

Another approach to improve the efficacy of chemotherapy may be the application of modifiers of chemoresistance. The heat-shock protein 90 (HSP90) is one of the most important proteins for tumor growth, resistance and survival. This protein makes up to 3\% of the whole protein content and is 2-3-fold upregulated in tumors compared to healthy tissue $[12,13]$. HSP90 is an ATP-dependent molecular chaperone which interacts with a broad range of different oncoproteins by forming up the HSP90 chaperone machinery together with HSP70 and additional co-chaperons. The abnormal high expression of HSP90 found in gastric cancer is taken into consideration as an independent prognostic marker [14, 15]. This machinery contributes to the regulation of proteins involved in the pathogenesis of gastric cancer: cell motility (e.g. epidermal growth factor receptor (EGFR), phosphoinositide-dependent protein kinase 1, c-Src), cell adhesion (e.g. focal adhesion kinase) and angiogenesis (e.g. vascular endothelial growth factor receptor; hypoxia-inducible factor-1) [16,17]. The HSP90 inhibitor ganetespib greatly affected EGFR signaling cascades in several gastric cancer cell lines and inhibited the growth of xenograft tumors in vivo as a single agent or in combination with cisplatin [16].

The HSP90 machinery plays, according to the literature, a major role in the stabilization of mutated p53 (mutp53). Frequently found missense mutations in p53 can lead to a tumor suppressor loss-of-function and possible even result in an oncogenic gain-of-function activity with an impact on progression, invasion metastasis and resistance to therapy. Therefore, prevention of mutp53 stabilization by the inhibition of HSP90 may be an attractive target in cancer treatment [18-20]. During our study, we focused on the evaluation of gastric cancer cell lines which differ in their type and p53 mutation status. MKN1 (primary adenosquamous) holds a p53 mutation at codon 143 (Val to Ala), while MKN28 (well-differentiated intestinal) holds one at codon 257 (Ile to Leu). MKN45 (poorly differentiated) does have a wild type p53 status and KATOIII cells (signet ring cell "diffuse type" carcinoma) show a genomic deletion of the gene [21]. Effects of chemotherapeutics and ganetespib were checked in cytotoxicity assays, Western blots and protein arrays.

\section{Materials and Methods}

\section{Cell lines and culture conditions}

Cell lines were obtained from the American and European Culture Collections ATCC and ECACC, respectively. Cells were cultured in RPMI-1640 medium (Seromed, Berlin, Germany) supplemented with $10 \%$ fetal bovine serum (Seromed) and antibiotics (Sigma-Aldrich, St.Louis, MO, USA). Oxoplatin was kindly provided by Dr. Zoser B. Salama, Consultancy, Ravensburg, Germany.

\section{Cytotoxicity assays}

Cytotoxicity was assessed using MTT assays and nine dilution steps of respective chemotherapeutics and ganetespib. In brief, $1 \times 10^{4}$ cells in $100 \mu 1$ medium were distributed to wells of 96-wells microtiter plates (TPP, Trasadingen Switzerland) and ten 2-fold dilutions of the chemotherapeutics were added in triplicate. Assays were at least performed in triplicate. The plates were incubated for four days under tissue 
culture conditions and viable cells detected using a modified MTT assay (EZ4U, Biomedica, Vienna, Austria). $\mathrm{IC}_{50}$ values were determined using Origin 9.1 software (OriginLab, Northampton, MA, USA). For the assessment of drug interactions, tests were performed comprising the individual drugs alone and in combination, followed by calculation of the combination indices (CI) using the Chou-Talalay method with help of the Compusyn software (ComboSyn, Inc. Paramus, NJ, USA).

\section{Cell cycle distribution}

Cell cycle distributions were determined by propidium iodide (PI) staining and flow cytometry. 1 $x 10^{6}$ cells per well were incubated with the respective compound in six-well plates for three days. Cells were harvested and fixed with $70 \%$ ethanol at $-20^{\circ} \mathrm{C}$ for 30 min and stained with $20 \mu \mathrm{g} / \mathrm{ml}$ PI and $5 \mu \mathrm{g} / \mathrm{ml}$ ribonuclease $\mathrm{A}$ in $0.05 \% \mathrm{NP} 40 / \mathrm{PBS}$. Cells were analyzed by flow cytometry (Cytomics FC500, Beckman Coulter, Brea, CA, USA) and MultiCycle AV software (Phoenix Flow Systems, San Diego, CA, USA).

\section{Western blot analysis}

Proteins were isolated using standard RIPA buffer protocols. $15 \mu \mathrm{g}$ of the proteins were separated on precast Mini-Protean $4-15 \%$ TGX gels at 250 Volts (Bio-Rad Laboratories, Hercules, CA, USA). Afterwards, the proteins were transferred on Trans-Blot Turbo 0,2 $\mu \mathrm{m}$ PVDF membranes via the Trans-Blot Turbo Transfer System and the preinstalled mixed molecular weight program (Biorad). The membranes were blocked with a 3\% non-fatty instant milk PBS/0.05\% Tween-20 solution under constant shaking. Antibody detection was performed using antibodies against p53 (BioLegend, San Diego, CA, USA), GAPDH (ProteinTech Europe, Manchester, UK) and CAIX (Novus Biologicals LLC, Littleton, CO, USA). HRP-conjugated secondary antibodies were purchased from Santa Cruz (Santa Cruz Biotechnology, Santa Cruz, CA, USA). Bands were visualized by using the WesternBright Chemiluminescence Substrate Sirius (Biozym Scientific $\mathrm{GmbH}$, Oldendorf, Germany) and the VersaDoc 4000MP Imaging System (Biorad).

\section{Proteome Profiler Array Kit}

Receptor tyrosine kinases (RTKs) were detected using a Western Blot array (Proteome Profiler Human Phospho-RTK Array Kit, ARY001B, R\&D Systems, Minneapolis, MN, USA) according to the manufacturer's instructions. The experiments were done in duplicate. In brief, $1 \times 10^{7}$ cells $/ \mathrm{ml}$ were washed in PBS and extracted using the appropriate buffer of the kit supplemented with protease inhibitor cocktail (Sigma-Aldrich). Extracts were centrifuged, the supernatants diluted with sample buffer and applied to the nitrocellulose membranes having 49 anti-kinase catcher antibodies spotted. Phosphorylation of RTKs was finally determined using pan-anti-tyrosine-HRP conjugate and detection of binding by chemiluminescence. Spots were analyzed using GelAnalyzer and ORIGIN 9.0 software (OriginLab, Northampton, MA, USA).

\section{Measurement of gene expression by qPCR}

Total RNA was isolated by using InnuSolv (Analytik Jena, Jena, GER) and $1 \mu \mathrm{g}$ of total RNA was reverse transcribed by using the High Capacity Reverse Transcription Kit from Thermo Fisher (Waltham, MA, USA). For qPCR analysis, $20 \mathrm{ng}$ of cDNA were mixed with $1 \mu \mathrm{l}$ primer (MUC1: Hs00159357_m1 (Thermo Fisher); geNorm gene selection kit (PrimerDesign, Southhampton, UK), 10 $\mu 1$ 2x TaqMan Gene Expression MasterMix (Thermo Fisher) and $7 \mu 1$ RNase/DNase free water in a 96-ell plate. qPCR was performed on the QuantStudio 12K Flex Real-Time PCR System (Thermo Fisher) with the following program: two minutes at $50^{\circ} \mathrm{C}$, two minutes at $95^{\circ} \mathrm{C}$ and 40 cycles of $95^{\circ} \mathrm{C}$ for 15 seconds and $60^{\circ} \mathrm{C}$ for one minute. Resulting Ct values were normalized with appropriate housekeeping genes and analyzed using the $2-\Delta \Delta \mathrm{Ct}$ method for relative gene expression determination.

\section{Statistics}

Statistical significance was tested by t-tests and $p<0.05$ regarded as significant difference.

\section{RESULTS}

\section{Expression of p53 and sensitivity to ganetespib by the gastric cancer cell lines}

Expression of p53 was tested in all four gastric cancer cell lines (fig.1A). As expected, the cell lines possessing mutp53, namely MKN1 and MKN28, showed high protein expression, whereas wildtype MKN45 showed very low expression and KATOIII proved to be negative for this tumor suppressor protein. To test the effect of ganetespib on p53 expression, MKN28 cells were preincubated with three different concentrations of this HSP90 inhibitor and p53 was analyzed using Western blots (fig.1B). Concentrations of ganetespib of 20, 200 and $2000 \mathrm{nM}$ did not change expression of p53 significantly. Similar results were obtained for the MKN1 cell line and for the low expression of wildtype p53 in MKN45 cells (data not shown). All four gastric cancer cell lines exhibited high and comparable expression of HSP90 (fig.1C). 

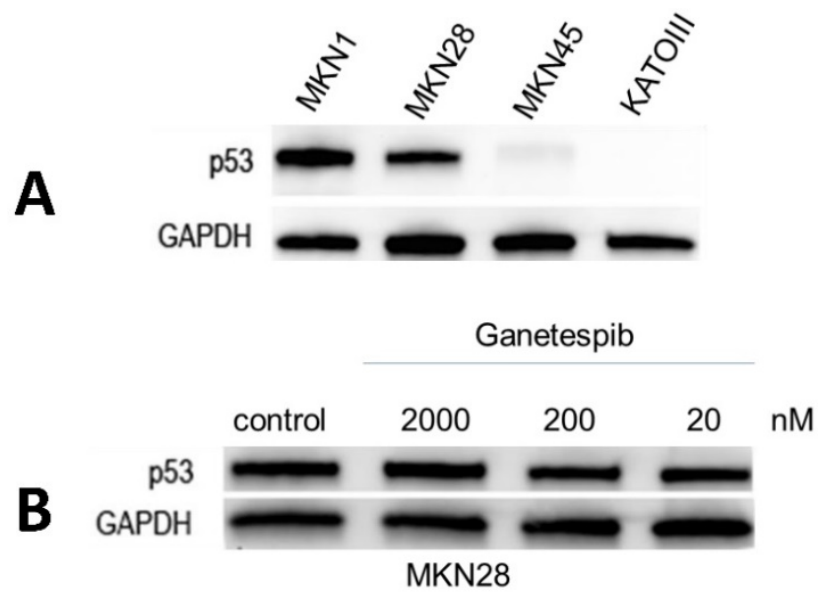

\begin{tabular}{llllll} 
& MKN1 & MKN28 & MKN45 & KATOIII \\
\cline { 3 - 4 } & & & & & \\
\hline & & & & &
\end{tabular}

Fig. 1: Expression of p53 and HSP90 by the gastric cancer cell lines and effect of ganetespib on p53 expression of MKN28. (A) p53 mutated cell lines MKN-1 and MKN-28 show high expression of $p 53$, while wild-type expression in MKN45 is barely detectable. No signal is detected in KATOIII cells with a genomic deletion of p53. (B) MKN-28 cells were preincubated with indicated concentrations of ganetespib and Western blots revealed no significant change in $\mathrm{p} 53$ protein level. (C) MKN-1, MKN-28, MKN-45 and KATOIII cells express comparable protein levels of HSP90.

\section{Cytotoxicity of ganetespib against the three MKN cell lines}

Cytotoxicity of ganetespib was tested in MTT assays against the three MKN cell lines (fig.2). MKN28 was found to be chemosensitive, in contrast to the MKN1 and MKN28 gastric cancer cell lines which exhibited $\mathrm{IC}_{50}$ values exceeding peak plasma concentrations of the drug by far (fig.2). Thus, chemosensitivity to ganetespib is not correlated with mutation status of p53 in the MKN cell lines. While ganetespib does not show a strong cytotoxic effect in MKN-1 and MKN-45 cell lines, the highly proliferative cell line MKN-28 was sensitive to the treatment with this HSP90 inhibitor. Ganetespib $\mathrm{IC}_{50}$ values were: $0.01 \pm 0.01 \mu \mathrm{M}$ for KATOIII, $1.76 \pm 0.44 \mu \mathrm{M}$ for $\mathrm{MKN}-1,0.18 \pm 0.26 \mu \mathrm{M}$ for MKN-28 and $0.45 \pm 0.26$ $\mu \mathrm{M}$ for $\mathrm{MKN}-45$, respectively. For comparison, the normal murine intestinal epithelial cell line IEC6 exhibited an $\mathrm{IC}_{50}$ value of $0.06 \pm 0.01 \mu \mathrm{M}$ for ganetespib.

Corresponding to the cytotoxic effects of ganetespib on the respective cell line, sensitive KATOIII and MKN28 cells exhibited a shift from S-phase towards a G2/M arrest. Insensitive MKN-1 cells do not show an altered cell cycle in response to ganetespib, while MKN-45 cells shifted cell cycle distribution towards G1/0 (data not shown).

\section{Chemosensitivity of the gastric cell lines to selected platinum compounds}

Chemosensitivity of the gastric cancer cell lines to platinum compounds were determined in dose-response curves employing MTT assays and after curve fitting yielded the results presented in table 1 . Whereas the parent compound oxoplatin is rather inert, DATCP[IV] and cisplatin are highly active against the gastric cancer cell lines.

Table 1. IC50 values $(\mu \mathrm{g} / \mathrm{ml})$ for the gastric cancer cell lines and the normal IEC6 line for the platinum compounds (mean values \pm SD).

\begin{tabular}{llllll}
\hline & KATOIII & MKN1 & MKN28 & MKN45 & IEC6 \\
\hline Oxoplatin & $9.54 \pm 0.80$ & $6.90 \pm 2.88$ & $10.00 \pm 2.5$ & $2.65 \pm 2.35$ & $>10$ \\
DATCP[IV] & $1.24 \pm 1.15$ & $2.72 \pm 1.86$ & $3.89 \pm 1.7$ & $1.43 \pm 4.45$ & $4.37 \pm 0.81$ \\
Cisplatin & $1.35 \pm 0.75$ & $1.28 \pm 0.54$ & $1.20 \pm 0.31$ & $1.82 \pm 1.22$ & $5.14 \pm 1.7$ \\
\hline
\end{tabular}

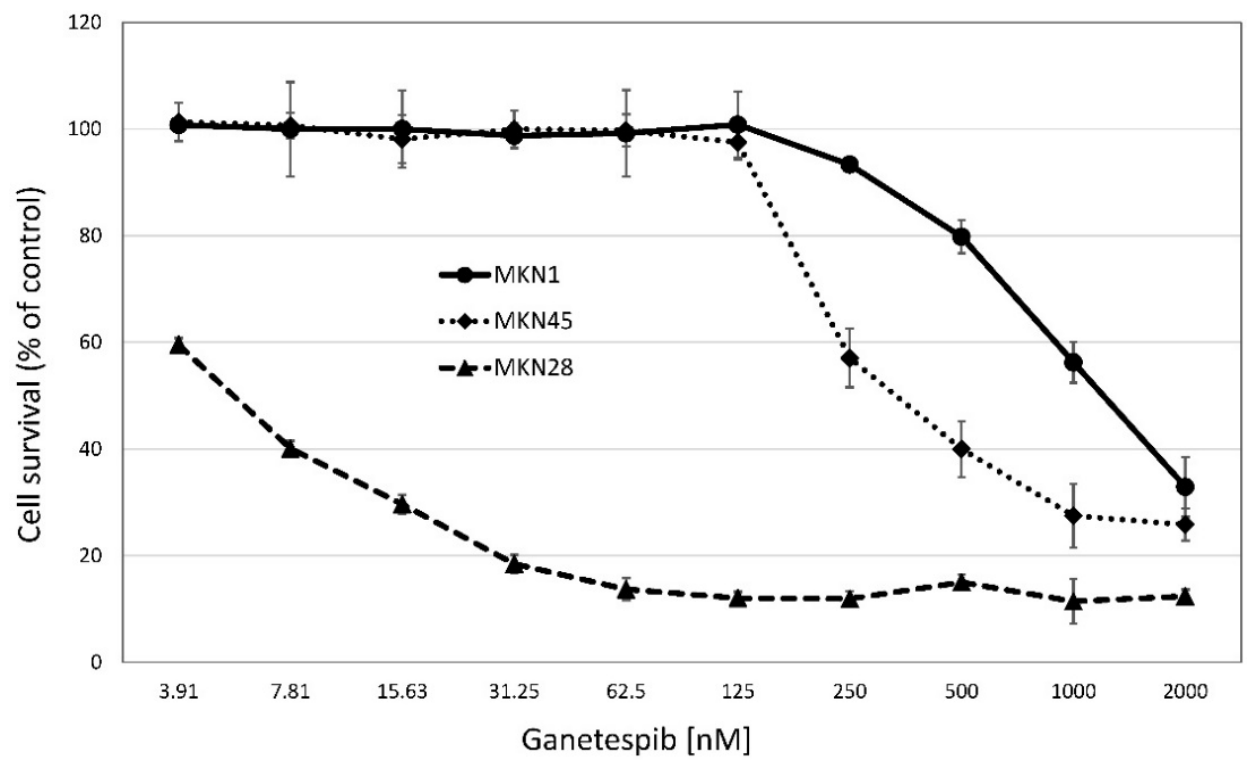

Fig.2. Cytotoxicity of ganetespib against MKN-1, MKN-28 and MKN-45 cell lines. Cell survival measurements are shown as mean values \pm SD. Anticancer activity of ganetespib was assessed using MTT assays with continuous presence of the drug for four days and $I_{50}$ values were calculated from dose-response curves covering a range of $3.8-2000 \mathrm{nM}$ of ganetespib. 


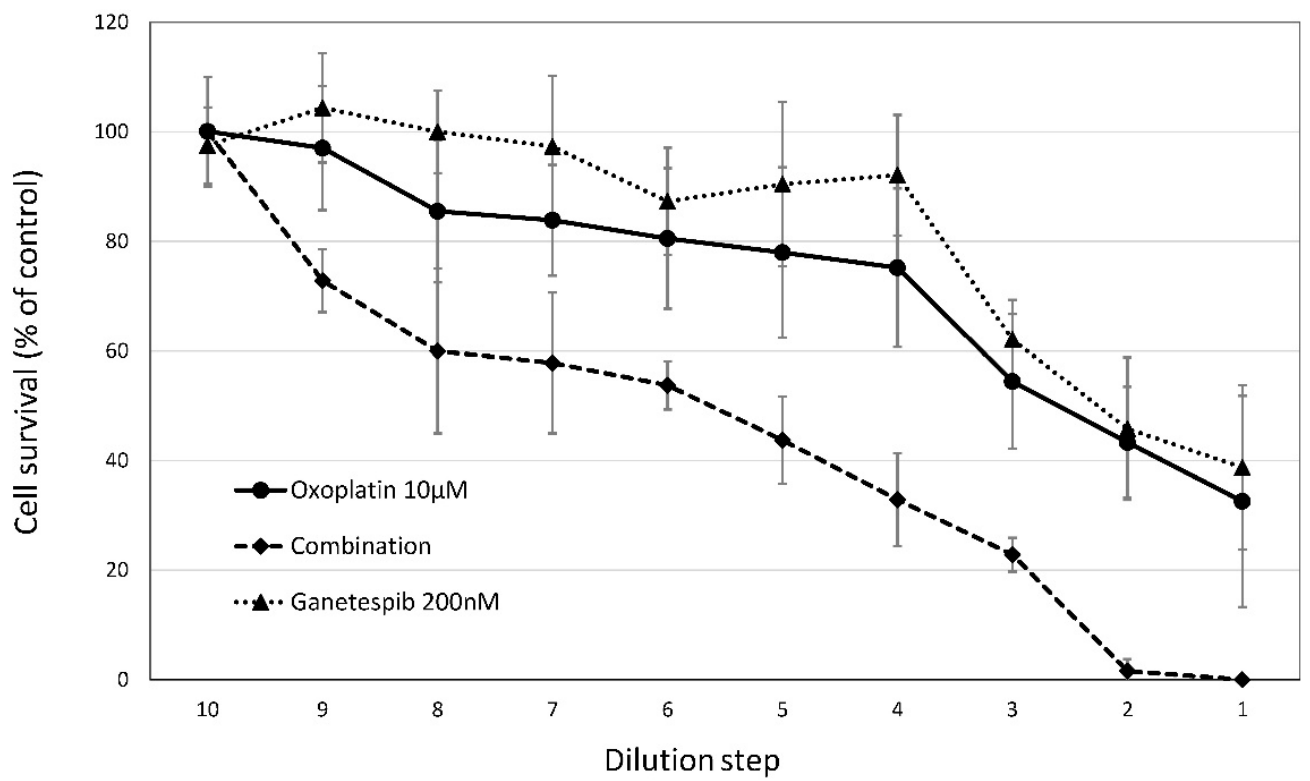

Fig. 3. Effects of cisplatin/oxoplatin in combination with ganetespib are shown for MKN-45 gastric cancer cell line. Cytotoxic effects of ganetespib were tested in combination with cisplatin or oxoplatin against the MKN-45 gastric cancer cell line. For the combinations, fixed ratios (initial concentrations were $10 \mu \mathrm{M}$ cis-/oxoplatin and $200 \mu \mathrm{M}$ ganetespib) of the platinum substances and ganetespib were diluted in twofold steps and cytotoxic activities were determined by MTT assays.

\section{Chemomodulatory effects of ganetespib on sensitivity of gastric cancer cell lines to platinum compounds}

The cytotoxic activities of oxoplatin and cisplatin against the gastric cancer cell lines were tested for a possible potentiation using the HSP90 inhibitor ganetespib. Combinational treatment resulted in a marked synergistic cytotoxic effect as shown in figure 3. for oxoplatin with ganetespib. The extent of chemosensitization effected by ganetespib is shown in table 2. Factors of 1.8 to 7.93 were observed, demonstrating an increased cytotoxicity of the platinum compounds in combination with the HSP90 inhibitor. A minor synergistic activity $(<1.15)$ was found for the combinations of platinum compounds and ganetespib in IEC6 cells.

Table 2. Chemosensitization of $M K N$ cell lines to platinum compounds by ganetespib shown in $\mathrm{IC}_{50}$ control/IC 50 ganetespib (mean values \pm SD) ratios.

\begin{tabular}{lll}
\hline \multicolumn{3}{c}{ Oxoplatin/Ganetespib } \\
\hline & Cisplatin/Ganetespib \\
MKN1 & $1.80 \pm 0.64$ & $2.05 \pm 1.06$ \\
MKN28 & $7.04 \pm 3.76$ & $2.52 \pm 0.58$ \\
MKN45 & $7.93 \pm 0.96$ & $3.50 \pm 1.74$ \\
\hline
\end{tabular}

\section{Concentration range of synergy for the platinum/ganetespib combinations}

Synergism between cytotoxic agents are often presented for one specific concentration of the combined drugs. However, for clinical application concentrations exhibiting synergism should cover the whole range applicable since in specific combinations this may be switched to antagonism. Figure 4 . presents the range of synergistic combinations (combination index CI $<1$ ) of the platinum compounds with ganetespib for each of the four gastric cancer cell lines, respectively.

\section{Effects of ganetespib on phosphorylation of kinases in MKN28 and MKN45}

For MKN28 the most significant effects of ganetespib were found for alterations of the phosphorylation of extracellular signal-regulated kinases 1/2 (ERK1/2), WNK Lysine Deficient Protein Kinase 1 (WNK1) and p53 (fig. 5A). For MKN45 the most significant effects of ganetespib were found to be changes of the phosphorylation of $40 \mathrm{KDa}$ Proline-Rich AKT Substrate (PRAS40), p70 56 kinase, WNK1, p53 and protein expression of HSP60 (fig. 5B).

\section{Effect of ganetespib on expression of CAIX and MUC1 in MKN28 and MKN45 cells}

RT-PCR experiments showed that expression of MUC1 is reduced at high levels of ganetespib (200 $\mathrm{nM}$ ) in MKN28 and MKN45 cells but not at concentrations achievable in the clinical setting (data not shown). In contrast, expression of CAIX which is a negative prognostic marker in gastric cancer is downregulated even at low concentrations in KATOIII and MKN45 cells. These cell lines were chosen because the exhibited the highest expression of CAIX. 


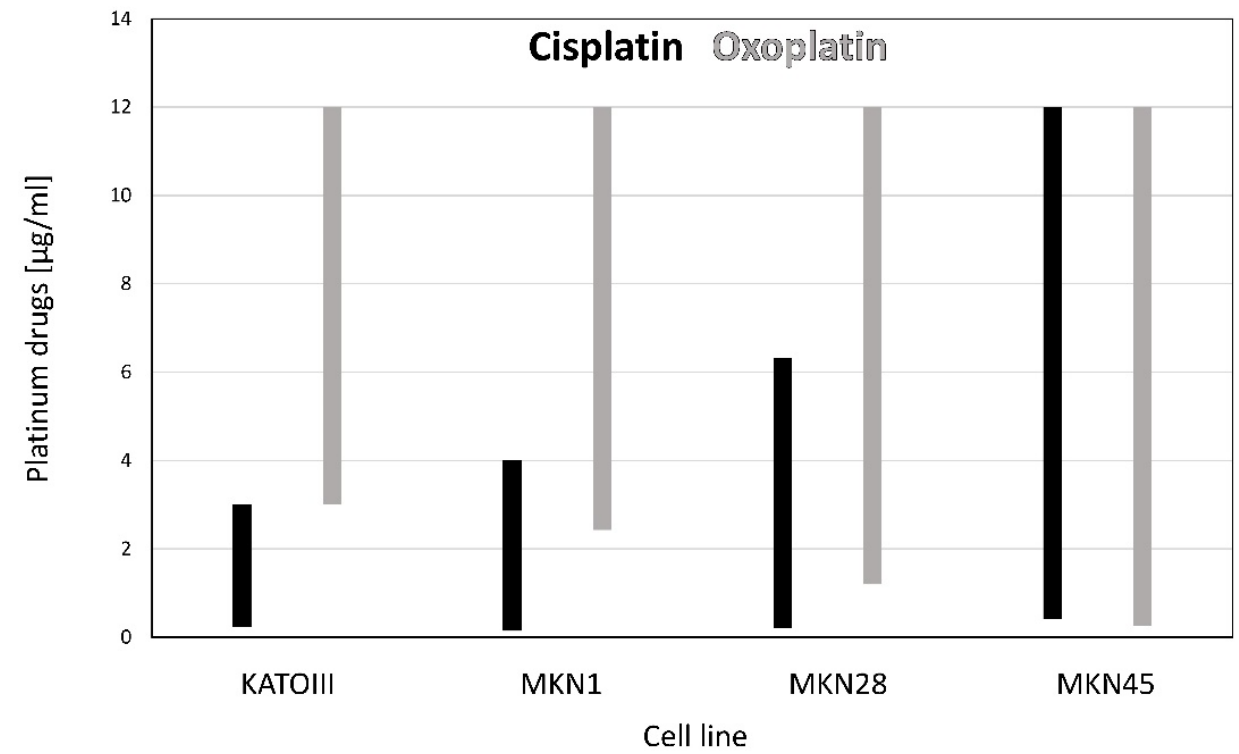

Fig. 4. Ranges of concentrations of chemotherapeutics which proved to exhibit synergism with ganetespib are depicted for the platinum drugs cisplatin and oxoplatin. The range of concentrations of platinum compounds which gave synergistic effects in combination with ganetespib (combination index $\mathrm{Cl}$ $<1$ ) is presented for the four gastric cancer cell lines. Cisplatin is depicted in black, oxoplatin in grey columns.

\section{DISCUSSION}

Gastric cancer is still the third leading cause of cancer death worldwide and, at advanced stage, is treated with chemotherapy since almost two-thirds of the gastric cancer patients are found to have unresectable disease or metastases at first presentation [1,7]. Additionally, up to $80 \%$ of patients develop locoregional or distant recurrence after an apparently curative resection. However, gastric cancer develops resistance to chemotherapeutic agents in a relatively short time and most patients would die within one year $[8,22]$. The introduction of agents targeting EGFR and the VEGF circuit for therapy of gastric cancer as precision therapy holds promise but may be not available for the majority of patients from economic reasons [23, 24]. The highest incidence of gastric cancer is in East Asia, Eastern Europe and parts of South and Central America where adenocarcinomas of the distal stomach are more prevalent [2]. 5-FU/platinum combinations, with or without an anthracycline, as well as irinotecan and docetaxel-containing combinations are reasonable current treatment options [22]. The addition of a third drug to a two-agent regimen increases the response rate with a modest survival improvement but at the expense of increased toxicity [23]. The ToGA trial showed that trastuzumab, a humanized monoclonal antibody against HER2, when combined with chemotherapy, was associated with extended overall survival and progression-free survival in people with HER2-positive advanced gastric cancers [24].

p53 mutation is one of the most prevalent genetic alterations in gastric cancer [25]. Missense mutations in p53 generate aberrant proteins with abrogated tumor suppressor functions that can also acquire oncogenic gain-of-function activities that promote malignant progression, invasion, metastasis and chemoresistance [20]. Mutp53 proteins undergo massive constitutive stabilization specifically in tumors by HSPs. The most prominent member, HSP90 accounts for 1-3\% of the total protein content in human cells and may play an important role on tumor invasion, metastasis and prognosis [15]. Abnormally high expression of HSP90 has been found in gastric cancer and been greatly considered as an independent prognostic marker of progression [15-17]. HSP90 chaperoning represents an important role in the stabilization of diverse oncogenes including HER2, mutp53, ALK, mutant EGFR, and others in tumor cells. HSP90 was targeted with inhibitors of which geldanamycin was the first available drug but proved to be too toxic for clinical application [26]. Ganetespib which exhibits a more favorable clinical profile inhibits HSP90 client binding, resulting in proteasomal degradation of the respective proteins/oncogenes (fig. 5). HSP90 inhibitors display preferential activity toward malignant or rapidly proliferating cells and have been found to persist in tumor cells for an extended period [27]. 
A

MKN28
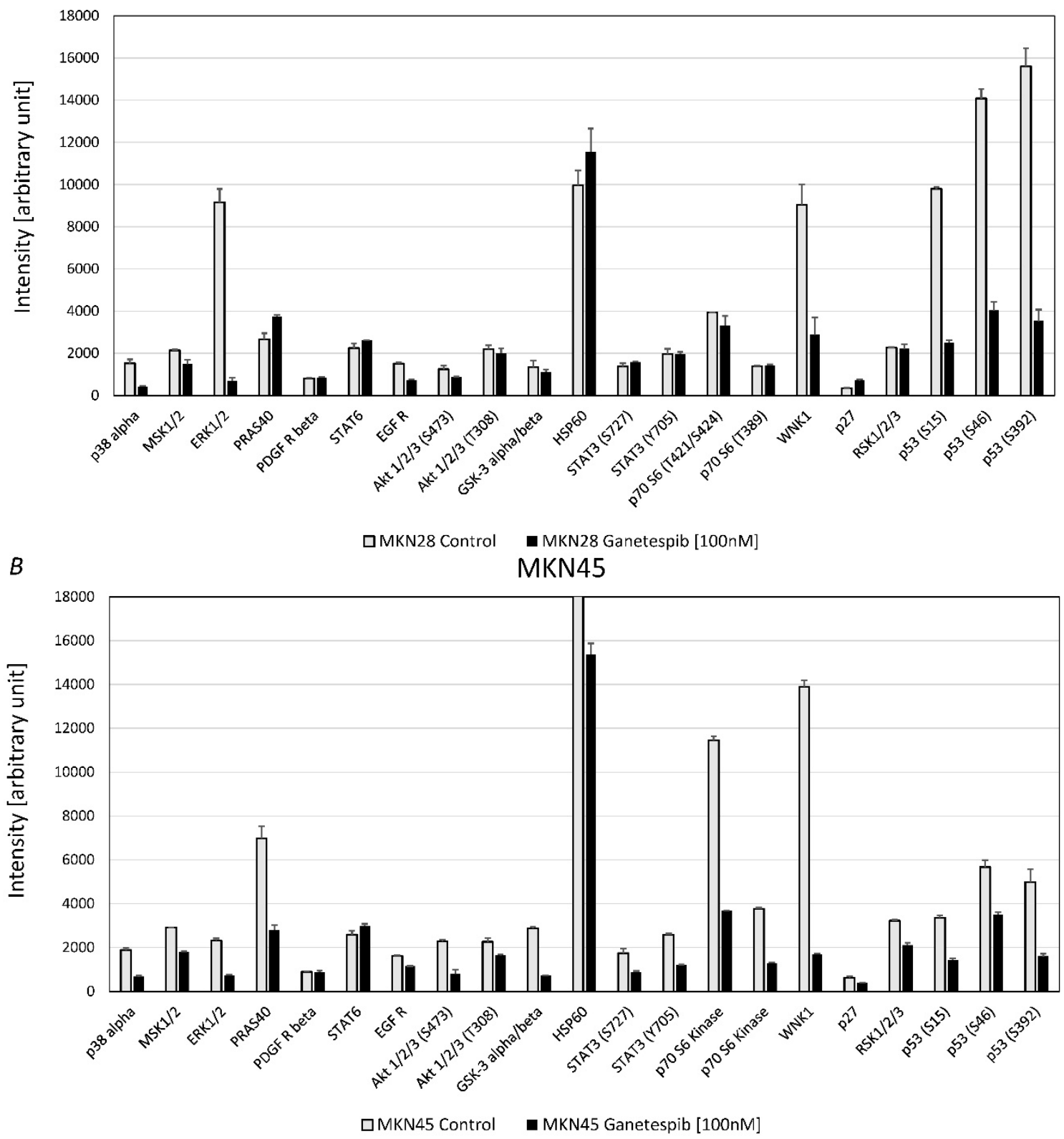

Fig. 5. A. Alterations of phosphoproteins in MKN28 in response to ganetespib treatment. Phosphorylation is shown as intensity (mean \pm SD) in pixel intensity units (except HSP60 protein expression). B. Alterations of phosphoproteins in MKN45 in response to ganetespib treatment. Phosphorylation is shown as intensity (mean \pm SD) in pixel intensity units (except HSP60 protein expression).

MKN1 adenosquamous primary tumor carcinoma line exhibits mutant p53 and silencing of E-cadherin and the MKN28 a well-differentiated intestinal type adenocarcinoma cell line shows mutations in p53 and APC tumor suppressor genes as well as silencing of CD44 [21]. The MKN-45 represents a differentiated diffuse type adenocarcinoma cell line with wild-type p53, deletion of p16CDKN2/ p15MTS2, amplification of c-met oncogene and mutation of E-cadherin. The KATO-III line exhibits genomic deletion of p53, amplification of c-met oncogene and mutation of E-cadherin and was not used in most experiments because it represents a p53-negative signet ring type tumor and this study aimed at characterization of the role of mutated p53. Ganetespib showed good cytotoxic effects in high proliferative gastric cancer cell lines, KATOIII and MKN-28, while less proliferative cell lines MKN-1 and MKN-45 showed resistance to this treatment. All investigated cell lines expressed comparable levels of HSP90 protein and, importantly, p53 protein levels did not change in response to ganetespib treatment. Cell cycle distribution shifted in sensitive cell lines from S-phase to G2/M arrest, while the cell cycle in the insensitive cell line MKN-1 was not affected. However, MKN-45 cells shifted towards an increased 
G1/0 arrest. With exception of strictly oncogene-addicted tumors, such as ALK-mutated NSCLC, HSP90 inhibition alone may not be sufficient to yield cytotoxic effects in advanced cancer [28]. Therefore, ganetespib in combination with other chemotherapeutic agents may be more efficient by enhancing their cytotoxicity and overcoming drug resistance. However, despite enormous promise and anticancer activity reported to date, none of the HSP90 inhibitors in development has been approved for cancer therapy [12]. Since platinum-based combinations are frequently used for the treatment of metastatic gastric cancer we tested cisplatin and a platinum (IV) derivative, namely oxoplatin, in combination with ganetespib for cytotoxic activity against the gastric cancer cell lines and normal IEC6 cells [9].
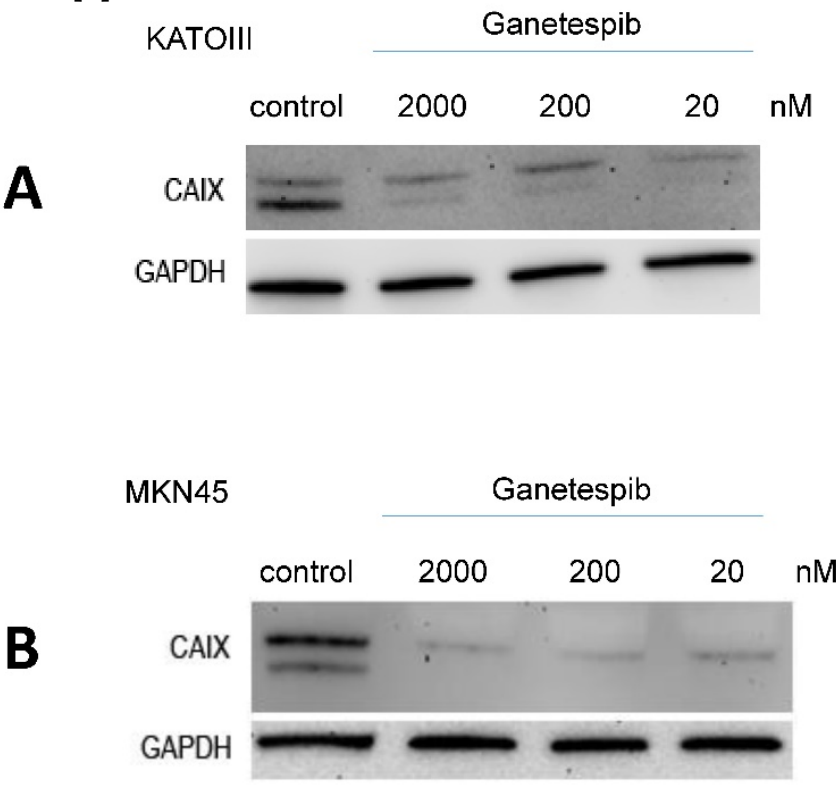

Fig. 6. Western blot detection of CAIX in KATOIII and MKN45 cells pretreated for 3 days with the indicated concentrations of ganetespib. Results are shown for KATO-III (A) and MKN-45 (B), respectively.

Combinational treatment of ganetespib with platinum substances, cis-/oxoplatin, resulted in a marked synergistic cytotoxic effect, except for the normal intestinal epithelial cell lines IEC6. Cisplatin (platinum(II)) must be administered as intravenous solution but platinum(IV) agents, such as oxoplatin, are considered to be largely inert prodrugs that are converted to highly cytotoxic platinum(II) compounds by reducing substances, enzymes or microenvironmental conditions. Reaction of oxoplatin with $0.1 \mathrm{M}$ hydrogen chloride mimicking gastric acid yields cis-diammine-tetrachlorido-platinum(IV) (DATCP[IV]), which exhibits at least two-fold increased cytotoxic activity [11]. Genome-wide expression profiling of a small cell lung cancer cell line treated with these platinum species revealed clear differences in the expression pattern of affected genes and cellular pathways between DATCP(IV) and cisplatin [29]. This effective synergistic cytotoxic effect in combination with the oral prodrug oxoplatin would be clearly superior for patient care compared to cisplatin. The normal IEC6 cells revealed low chemosensitivity to all three platinum compounds used in the current study, such suggesting a favorable toxicity profile of these drugs in the gastrointestinal tract. Currently, no oral platinum-based drug has been approved.

These results correspond to previous reports investigating HSP90 and gastric cancer cell lines. In four gastric cancer cells (MKN-1, MKN-7, MKN-45, and NUGC-4), 17-dimethylaminoethylamino-17demethoxygeldanamycin (17-DMAG) synergistically potentiated the effect of cisplatin on inhibition of cell growth [30]. Furthermore, ganetespib markedly reduced the growth of MGC-803 and significantly inhibited the growth of SGC-7901 and MKN-28 gastric cancer cells [16, 31]. Ganetespib caused pronounced decrease of expression of classic HSP90 client proteins, such as EGFR and, furthermore, ganetespib worked synergistically with radiation and cisplatin in cytotoxicity. Additionally, ganetespib significantly inhibited xenograft tumors in vivo as single agent or combined with cisplatin. Another HSP90 inhibitor, namely LD053, significantly inhibited growth of gastric cancer BGC823 xenografts [16, 31]. In a human ovarian cancer cell line, the expression of LRP, GST-ח, p53, bcl-2, survivin, ERCC1, XRCC1, BRCA1 and BRCA2 was greater impaired by HSP90 inhibitors in combination with cisplatin compared to HSP90 inhibitors or cisplatin treatment alone [32].

In a broader approach to identify the effects of ganetespib on signal transduction in the gastric cancer cell lines we used Western blot phosphokinase arrays to detect alterations in protein phosphorylation in MKN28 and MKN45 exposed to the HSP90 inhibitor. Arrays showed significant downregulation of three phosphorylation sites of p53 in both cell lines. Of course, wildtype p53 MKN45 cells express lower amounts of the protein in contrast to Mutp53 MKN28 cells. The residues supposed critical for p53 antiproliferative and antiapoptotic activities, including Ser15, Thr18, Ser20, Ser46 and Ser392, are infrequently mutated in cancers [33]. Minamoto et al. found that Mut53 can be phosphorylated at various sites including Ser15, Thr81 and Ser392 in vitro and in human tumors in vivo [34]. Other studies have reported a correlation between a high rate of cells with hyperphosphorylation of Ser392 and poor prognosis or advanced tumor stage in p53-positive cancers including esophageal squamous cell 
carcinomas and a variety of skin tumors [35-37]. The expression of approximately 3\% of genes was altered in both 5-FU- and cisplatin-resistant gastric cancer p53 wild-type AGS cells with highest alterations of genes associated with the p53-associated pathways [38]. S392 phosphorylation of p53 may enhance the tetramer formation of certain gain-of-function mutants, yielding potent oncoproteins. In conclusion, reduced phosphorylation of p53 by ganetespib may reduce the malignity of tumor cells.

EGFR has been implicated in gastric cancer growth and constitutes a therapeutic target. Upon ligand binding, EGFR, generating binding sites for mediators that stimulate proliferation and chemotaxis via the PI3K/AKT/mTOR pathway [39]. A strong positive relation was detected between pEGFR and MMP7/13 in gastric cancer [40]. HSP90 inhibition by AUY922 treatment deactivated PI3K/AKT as well as Raf/MAPK/ERK pathways in three liposarcoma cell lines [41]. Furthermore, macrophages stimulate gastric cancer invasion through phosphorylation of EGFR Y1086, c-Src, Erk1/2 and Akt phosphorylation [42]. However, the Western blot arrays showed low activity of EGFR in MKN28 and MKN45 cell lines, although the downstream targets ERK1/2 are hyperphosphorylated in MKN28.

Trastuzumab, a humanized antibody targeting HER2, exhibits efficacy against receptor-positive gastric cancer with Akt signaling pathway involved in chemoresistance [17]. Ganetespib downregulates the Akt1/2/3 S473 phosphorylation and may thus help to reduce resistance to trastuzumab [18]. Another protein with markedly reduced phosphorylation in MKN45 is the proline-rich AKT substrate of $40-\mathrm{kDa}$ (PRAS40) which inhibits the activity of the mTOR complex 1 (mTORC1) kinase. Phosphorylation of PRAS40 by Akt and by mTORC1 itself results in activation of mTORC1 activity and enhancement of cell survival and growth via activation of $\mathrm{p}-70 \mathrm{S6K}$ and 4-EBP1 [43-45]. Accordingly, phosphorylation of 70S6K und Akt is downregulated in MKN45 cells in response to ganetespib. More than half of the primary gastric cancers demonstrated positive phospho-PRAS40 (Thr246) expression, which correlates with lymph node metastasis and venous invasion [44]. PRAS40 inhibition by ganetespib was previously reported in inflammatory breast cancer cell line SUM149 [46].

The most significant effect on kinase phosphorylation in both MKN28 and MKN45 cell lines affected the serine/threonine protein kinase WNK1 (with no lysine (K)1). This kinase plays an important role in the regulation of electrolyte homeostasis, cell signaling, survival, and proliferation as an activator and inhibitor of sodium-coupled chloride cotransporters and potassium-coupled chloride cotransporters, respectively [47, 48]. The reduction of intracellular chloride concentration was reported to inhibit the proliferation of MKN28 gastric cancer cells through upregulation cyclin-dependent kinase inhibitor p21 [49]. Cultivation of MKN28 cells in a low chloride medium significantly induced phosphorylation of MAPKs (ERK, p38, and JNK) and G1/S cell-cycle arrest [49]. Furthermore, cell-cycle arrest was associated with downregulation of CDK2 and phosphorylated retinoblastoma protein without any effects on p53 [50]. Activation of the WNK1/OSR1/NKCC1 signaling pathway was demonstrated to enhance glioma migration in response to temozolomide [51].

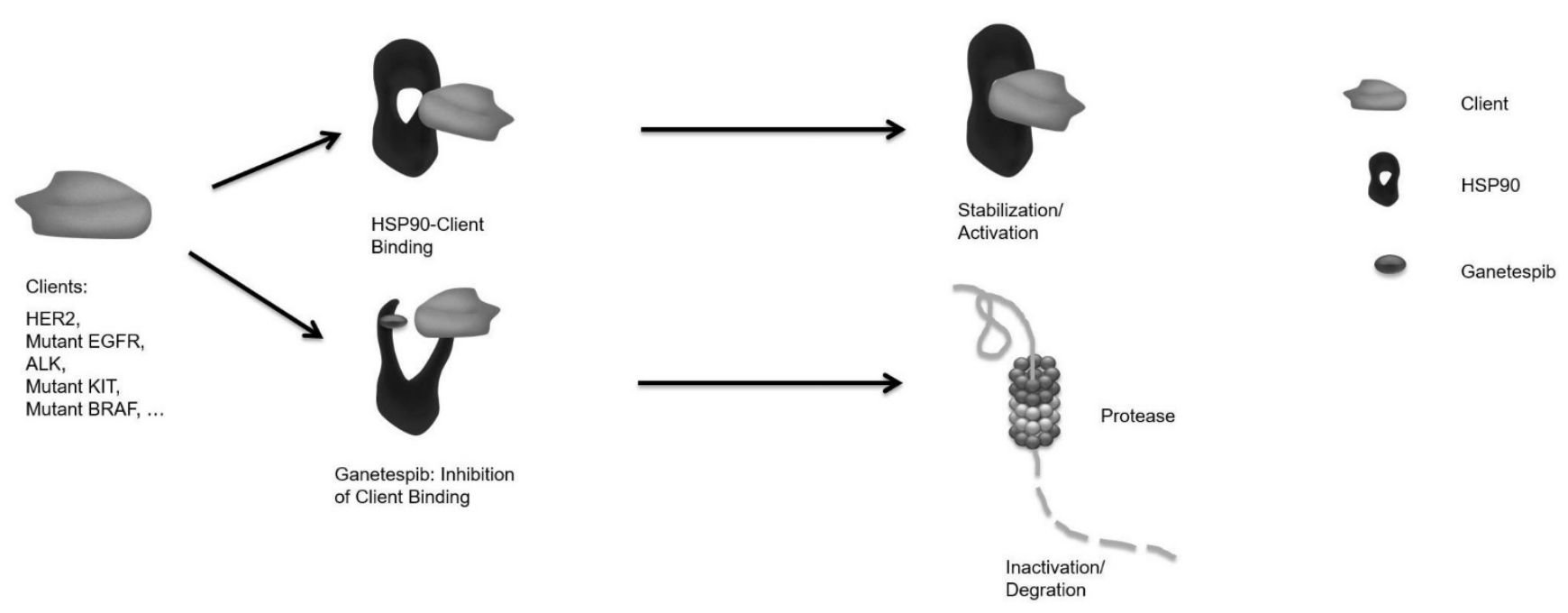

Fig. 7. Schematic presentation of the cellular function of HSP9O. 
HSP60, together with its associated chaperonin, HSP10, is a stress response chaperone which counteracts apoptosis by prevention of loss of mitochondrial membrane potential and translocation of cytochrome c [52]. HSP60 protein was significantly associated with invasion, metastasis and stage of disease as independent prognostic factor in gastric cancer [53]. In the present study, HSP60 protein is expressed at high levels in both MKN28 and MKN45 cells and resistant to treatment with ganetespib. Expression of two other proteins, namely MUC1 and CAIX, was tested after treatment of KATOIII and MKN45 with ganetespib. Whereas MUC1 expression was not changed in response to $20 \mathrm{nM}$ ganetespib, expression of CAIX was abolished in reaction to 20 $-2000 \mathrm{nM}$ of this HSP90 inhibitor. MUC1 is a glycoprotein with extensive O-linked glycosylation protect epithelial gastric cells but is a prognostic marker of a dismal prognosis in gastric cancer patients [54, 55]. Carbonic anhydrases (CAs) convert carbon dioxide to bicarbonate and protons and targeting the tumor associated isoform CAIX is tried as therapeutic modality against hypoxic and invasive tumors [56, 57]. CAIX expression is an independent prognostic factor for overall survival and metastasis-free survival in esophageal and gastric adenocarcinomas [58,59]. A regulation of the expression of CAIX by ganetespib was not reported so far.

In conclusion, ganetespib exhibits anticancer activity through several targets in the gastric cancer cell lines. In contrast to other tumor types, the amount of p53 is not changed but p53 phosphorylation is reduced in wildtype and mutated forms. In addition to the partial reversal of cisplatin resistance, this HSP90 inhibitor downregulates phosphorylation of Akt1/2/3 and WNK1 and expression of CAIX. Thus, the oral platinum compound oxoplatin represents a promising agent for the treatment of gastric cancer, especially in combination with the chemosensitizing HSP90 inhibitor ganetespib which regulates other proteins involved in tumor aggressiveness [60].

\section{Competing Interests}

The authors have declared that no competing interest exists.

\section{References}

1. Ferlay J, Soerjomataram I, Dikshit R, Eser S, Mathers C, Rebelo M, et al. Cancer incidence and mortality worldwide: Sources, methods and major patterns in GLOBOCAN 2012. Int J Cancer. 2015; 136: E359-86.

2. Roder DM. The epidemiology of gastric cancer. Gastric Cancer. 2002; 5(S1): 5-11.

3. Uemura N, Okamoto S, Yamamoto S, Matsumura N, Yamaguchi S, Yamakido $\mathrm{M}$, et al. Helicobacter pylori infection and the development of gastric cancer. $\mathrm{N}$ Engl J Med. 2001; 345:784-9.

4. Guggenheim DE, Shah MA. Gastric cancer epidemiology and risk factors. J Surg Oncol. 2013; 107: 230-6.
5. Zang ZJ, Cutcutache I, Poon SL, Zhang SL, McPherson JR, Tao J, et al. Exome sequencing of gastric adenocarcinoma identifies recurrent somatic mutations in cell adhesion and chromatin remodeling genes. Nature Genet. 2012; 44: 570-4.

6. Allum WH, Blazeby JM, Griffin SM, Cunningham D, Jankowski JA, Wong R. Guidelines for the management of oesophageal and gastric cancer. Gut. 2011; 60: 1449-72.

7. Choi AH, Kim J, Chao J. Perioperative chemotherapy for resectable gastric cancer: MAGIC and beyond. World J Gastroenterol. 2015; 21: 7343-8.

8. Wagner AD, Unverzagt S, Grothe W, Kleber G, Grothey A, Haerting J, et al. Chemotherapy for advanced gastric cancer. Cochrane Database Syst Rev. 2010; 3: CD004064.

9. Olszewski U, Ach F, Ulsperger E, Baumgartner G, Zeillinger R, Bednarski P, et al. In Vitro Evaluation of Oxoplatin: An Oral Platinum(IV) Anticancer Agent. Metal-Based Drugs. 2009; 2009:348916.

10. Wexselblatt E, Gibson D. What do we know about the reduction of $\mathrm{Pt}(\mathrm{IV})$ pro drugs? J Inorg Biochem. 2012; 117: 220-9.

11. Hamilton G. Comparison of Intracellular Stress Response of NCI-H526 Small Cell Lung Cancer (SCLC) Cells to Platinum(II) Cisplatin and Platinum(IV) Oxoplatin. Cancers (Basel). 2014; 6: 1487-99.

12. Barrot JJ, Haystead TAJ. Hsp90. An unlikely ally in the war on cancer. FEBS J. 2013; 280: 1381-96

13. Moulick K, Ahn JH, Zong H, Rodina A, Cerchietti L, Gomes EM, et al. Affinity-based proteomics reveal cancer-specific networks coordinated by Hsp90. Nat Chem Biol. 2011; 7: 818-26.

14. Pratt $\mathrm{WB}$, Toft DO. Regulation of signaling protein function and trafficking by the hsp90/hsp70-based chaperone machinery. Exp Biol Med (Maywood). 2003; 228: 111-33.

15. Wang J, Cui S, Zhang X, Wu Y, Tang H. High expression of Heat Shock Protein 90 Is Associated with Tumor Aggressiveness and Poor Prognosis in Patients with Advanced Gastric Cancer. PLoS One. 2013; 8: e62876.

16. Liu H, Lu J, Hua Y, Zhang P, Liang ZRuan L, et al. Targeting heat-shock protein 90 with ganetespib for molecularly targeted therapy of gastric cancer. Cell Death and Disease. 2015; 6: e1595.

17. Neckers L, Workman P. Hsp90 Molecular Chaperone Inhibitors: Are We There Yet? Clin Cancer Res. 2012; 18: 64-76.

18. Hanel W, Marchenko N, Xu S, Yu SX, Weng W, Moll U. Two hot spot mutant p53 mouse models display differential gain of function in tumorigenesis. Cell Death Diff. 2013; 20: 898-909.

19. Lang GA, Iwakuma T, Suh YA, Liu G, Rao VA, Parant JM, et al. Gain of function of a p53 hot spot mutation in a mouse model of Li-Fraumeni syndrome. Cell. 2004; 119: 861-72.

20. Alexandrova EM, Yallowitz AR, Li D, Xu S, Schulz R, Proia DA, et al. Improving survival by exploiting tumour dependence on stabilized mutant p53 for treatment. Nature. 2015; 523: 352-6.

21. Yokozaki H. Molecular characteristics of eight gastric cancer cell lines established in Japan. Pathology International. 2000; 50: 767-77.

22. Van Cutsem E, Sagaert X, Topal B, Haustermans K, Prenen H. Gastric cancer. Lancet. 2016; 388: 2654-64.

23. Jou E, Rajdev L. Current and emerging therapies in unresectable and recurrent gastric cancer. World J Gastroenterol. 2016; 22: 4812-23.

24. Bang YJ, Van Cutsem E, Feyereislova A, Chung HC, Shen L, Sawaki A, et all. Trastuzumab in combination with chemotherapy versus chemotherapy alone for treatment of HER2-positive advanced gastric or gastro-oesophageal junction cancer (ToGA): a phase 3, open-label, randomised controlled trial. Lancet. 2010; 376: 687-97.

25. Busuttil RA, Zapparoli GV, Haupt S, Fennell C, Wong SQ, Pang JM, et all. Role of p53 in the progression of gastric cancer. Oncotarget. 2014; 5: 12016-26.

26. Franke J, Eichner S, Zeilinger C, Kirschning A. Targeting heat-shock-protein 90 (Hsp90) by natural products: geldanamycin, a show case in cancer therapy. Nat Prod Rep. 2013; 30: 1299-323.

27. Prince TL, Kijima T, Tatokoro $\mathrm{M}$, et al. Client Proteins and Small Molecule Inhibitors Display Distinct Binding Preferences for Constitutive and Stress-Induced HSP90 Isoforms and Their Conformationally Restricted Mutants. PLoS One. 2015; 10: e0141786.

28. Thakur MK, Heilbrun LK, Sheng S, et al. A phase II trial of ganetespib, a heat shock protein $90 \mathrm{Hsp} 90$ ) inhibitor, in patients with docetaxel-pretreated metastatic castrate-resistant prostate cancer (CRPC)-a prostate cancer clinical trials consortium (PCCTC) study. Invest New Drugs. 2016; 34: 112-8.

29. Olszewski U, Ulsperger E, Geissler K, et al. Comparison of the effects of the oral anticancer platinum(IV) complexes oxoplatin and metabolite cis-diammine-tetrachlorido-platinum(IV) on global gene expression of NCI-H526 cells. J Exp Pharmacol. 2011; 3: 43-50.

30. Dote $H$, Hato S, Koshimune R, Ino H, Naito M, Date H. Synergic antiproliferative effect of Hsp90 inhibitor in combination with cisplatin in gastric carcinoma cell lines. Europ J Cancer. 2007; 5: 77 (abstract 374)

31. Liu H, Lu J, Hua Y, et al. Targeting heat-shock protein 90 with ganetespib for molecularly targeted therapy of gastric cancer. Cell Death Dis. 2015; 6: e1595.

32. Lu C, Liu D, Jin J, et al. Inhibition of gastric tumor growth by a novel Hsp90 inhibitor. Biochem Pharmacol. 2013; 85: 1246-56.

33. Nguyen TA, Menendez D, Resnick MA, Anderson CW. Mutant TP53 posttranslational modifications: challenges and opportunities. Hum Mutat. 2014; 35: 738-55. 
34. Minamoto $\mathrm{T}$, Buschmann $\mathrm{T}$, Habelhah $\mathrm{H}$, Matusevich $\mathrm{E}$, Tahara $\mathrm{H}$, Boerresen-Dale AL, Harris C, Sidransky D, Ronai Z. Distinct pattern of p53 phosphorylation in human tumors. Oncogene. 2001; 20: 3341-7.

35. Bar JK, Słomska I, Rabczyńki J, Noga L, Gryboś M. Expression of p53 protein phosphorylated at serine 20 and serine 392 in malignant and benign ovarian neoplasms: correlation with clinicopathological parameters of tumors. Int J Gynecol Cancer. 2009; 19: 1322-8.

36. Matsumoto M, Furihata M, Kurabayashi A, Sasaguri S, Araki K, Hayashi H, Ohtsuki Y. Prognostic significance of serine 392 phosphorylation in overexpressed p53 protein in human esophageal squamous cell carcinoma. Oncology. 2004; 67: 143-50.

37. Dai $\mathrm{C}, \mathrm{Gu} \mathrm{W}$. p53 post-translational modification: deregulated in tumorigenesis. Trends Mol Med. 2010; 16: 528-36.

38. Maeda O, Ando T, Ohmiya N, et al. Alteration of gene expression and DNA methylation in drug-resistant gastric cancer. Oncol Rep. 2014; 31: 1883-90.

39. Yamaoka T, Yan F, Cao H, Hobbs SS, Dise RS, Tong W, Polk DB. Transactivation of EGF receptor and ErbB2 protects intestinal epithelial cells from TNF-induced apoptosis. Proc Natl Acad Sci U S A. 2008; 105: 11772-7.

40. Ye Y, Zhou X, Li X, Tang Y, Sun Y, Fang J. Inhibition of epidermal growth factor receptor signaling prohibits metastasis of gastric cancer via downregulation of MMP7 and MMP13. Tumour Biol. 2014; 35: 10891-6.

41. Steinmann S, Gali-Muhtasib H, Huebner K, Al-Halabi R, Abou Merhi R, Aman $P$, et al. Hsp90 inhibition by AUY922 as an effective treatment strategy against myxoid liposarcoma. Cancer Lett. 2015; 367: 147-56.

42. Cardoso AP, Pinto ML, Pinto AT, Oliveira MI, Pinto MT, Gonçalves R, et all. Macrophages stimulate gastric and colorectal cancer invasion through EGFR $\mathrm{Y}(1086), \mathrm{c}-\mathrm{Src}$, Erk1/2 and Akt phosphorylation and small GTPase activity. Oncogene. 2014; 33: 2123-33.

43. Malla R, Ashby CR Jr, Narayanan NK, Narayanan B, Faridi JS, Tiwari AK. Proline-rich AKT substrate of 40-kDa (PRAS40) in the pathophysiology of cancer. Biochem Biophys Res Commun. 2015; 463: 161-6.

44. Lu YZ, Deng AM, Li LH, Liu GY, Wu GY. Prognostic role of phospho-PRAS40 (Thr246) expression in gastric cancer. Arch Med Sci. 2014; 10: 149-53.

45. Lai KP, Leong WF, Chau JF, Jia D, Zeng L, Liu H, et all. S6K1 is a multifaceted regulator of $\mathrm{Mdm} 2$ that connects nutrient status and DNA damage response. EMBO J. 2010; 29: 2994-3006.

46. Friedland JC, Smith DL, Sang J, Acquaviva J, He S, Zhang C, et all. Targeted inhibition of Hsp90 by ganetespib is effective across a broad spectrum of breast cancer subtypes. Invest New Drugs. 2014; 32: 14-24.

47. McCormick JA, Ellison DH. The WNKs: atypical protein kinases with pleiotropic actions. Physiol Rev. 2011; 91: 177-219.

48. de Los Heros P, Alessi DR, Gourlay R, Campbell DG, Deak M, Macartney TJ, et all. The WNK-regulated SPAK/OSR1 kinases directly phosphorylate and inhibit the K+-Cl- co-transporters. Biochem J. 2014; 458: 559-73.

49. Ohsawa R, Miyazaki H, Niisato N, Shiozaki A, Iwasaki Y, Otsuji E, Marunaka $Y$. Intracellular chloride regulates cell proliferation through the activation of stress-activated protein kinases in MKN28 human gastric cancer cells. J Cell Physiol. 2010; 223: 764-70.

50. Miyazaki H, Shiozaki A, Niisato N, Ohsawa R, Itoi H, Ueda $Y$, et all. Chloride ions control the G1/S cell-cycle checkpoint by regulating the expression of p21 through a p53-independent pathway in human gastric cancer cells. Biochem Biophys Res Commun. 2008; 366: 506-12.

51. Zhu W, Begum G, Pointer K, Clark PA, Yang SS, Lin SH, et al. WNK1-OSR1 kinase-mediated phospho-activation of $\mathrm{Na}+-\mathrm{K}+-2 \mathrm{Cl}$ - cotransporter facilitates glioma migration. Mol Cancer. 2014; 13: 31.

52. Xiao-shan Li, Qing Xu, Xiang-yang Fu, Wei-sheng Luo. Heat Shock Protein 60 Overexpression Is Associated with the Progression and Prognosis in Gastric Cancer. PLoS One. 2014; 9: e107507.

53. Li XS, Xu O, Fu XY, Luo WS. Heat shock protein 60 overexpression is associated with the progression and prognosis in gastric cancer. PLoS One. 2014; 9: e107507.

54. Wang XT, Kong FB, Mai W, Li L, Pang LM. MUC1 Immunohistochemical Expression as a Prognostic Factor in Gastric Cancer: Meta-Analysis. Dis Markers. 2016; 2016: 9421571.

55. Hollingsworth MA, Swanson BJ. Mucins in cancer: protection and control of the cell surface. Nat Rev Cancer. 2004; 4: 45-60.

56. Supuran CT, Winum JY. Carbonic anhydrase IX inhibitors in cancer therapy: an update. Future Med Chem. 2015; 7: 1407-14.

57. Chen J, Röcken C, Hoffmann J, Krüger S, Lendeckel U, Rocco A, et al. Expression of carbonic anhydrase 9 at the invasion front of gastric cancers. Gut. 2005; 54: 920-7.

58. Driessen A, Landuyt W, Pastorekova S, Moons J, Goethals L, Haustermans K, et al. Expression of carbonic anhydrase IX (CA IX), a hypoxia-related protein, rather than vascular-endothelial growth factor (VEGF), a pro-angiogenic factor, correlates with an extremely poor prognosis in esophageal and gastric adenocarcinomas. Ann Surg. 2006; 243: 334-40.

59. Griffiths EA, Pritchard SA, Welch IM, Price PM, West CM. Is the hypoxia-inducible factor pathway important in gastric cancer? Eur J Cancer. 2005; 41: 2792-805.

60. Garg G, Khandelwal A, Blagg BS. Anticancer Inhibitors of Hsp90 Function: Beyond the Usual Suspects. Adv Cancer Res. 2016; 129: 51-88. 